\title{
Dowling-Degos Disease Presenting Primarily with Comedones and Atrophic Scarring
}

\author{
Bianca M. Tod ${ }^{a} \quad$ Ilana Steenkamp ${ }^{b}$ Henry Francois Jordaan ${ }^{a}$ Willem I. Visser $^{a}$ \\ a Division of Dermatology, Faculty of Medicine and Health Sciences, Tygerberg Academic Hospital \\ and Stellenbosch University, Cape Town, South Africa; ${ }^{b}$ Division of Dermatology, Faculty of \\ Medicine, University of Cape Town, Cape Town, South Africa
}

\author{
Keywords \\ Comedones $\cdot$ Atrophic scarring $\cdot$ Reticulated pigmentary disorders
}

\section{Abstract}

Dowling-Degos disease (DDD) is a rare genodermatosis primarily presenting with reticulated pigmentation of the flexures. Secondary features include comedones and atrophic scarring. We present a patient with histologically confirmed DDD whose predominant clinical finding was of comedones and scarring, with less prominent pigmentation, thus expanding the clinical spectrum of DDD.

\section{Introduction}

Dowling-Degos disease (DDD) is an autosomal dominant genodermatosis presenting primarily with reticulated pigmentation of the flexures [1]. Secondary features of the disease include pitted facial and perioral scars and comedo-like lesions [2]. DDD exists within a spectrum of related conditions presenting with reticulated pigmentation, including Galli-Galli and Kitamura diseases [3]. The histology of this group of conditions is considered diagnostic [1]. The histology in this case made the diagnosis. 
Tod et al.: Dowling-Degos Disease Presenting Primarily with Comedones and Atrophic Scarring

Fig. 1. Comedo-like openings and atrophic scarring on the face.

Fig. 2. Blue-grey pigmentation and hidradenitis suppurativa in the left axilla.

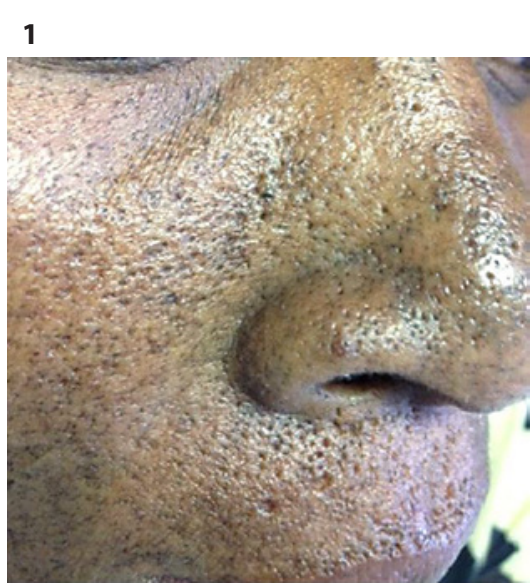

2

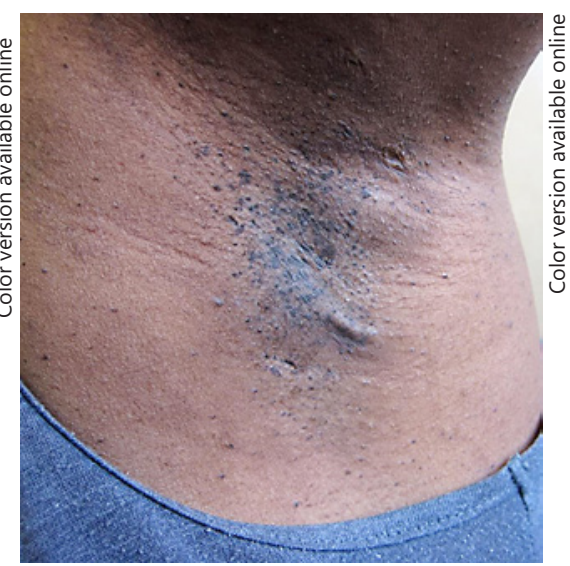

\section{Case Report}

A 36-year-old female presented with large, open comedo-like lesions on her face, chest, and back which had been present for approximately 3 years. She also complained of atrophic, pitted scars on her face (Fig. 1). She gave no history of preceding acne. She was known to be HIV positive and was being treated with antiretroviral medication. No association between timing of the appearance of the skin lesions and the initiation of the medication was noted. She gave no family history of similar lesions.

On examination, besides the aforementioned comedo-like lesions and scarring, subtle macular pigmentation was noted on the face, chest, and back, as well as more striking pigmentation in the axillae. The pigmentation in the axillae had a blue-grey color (Fig. 2). In some areas, the pigmentation was reticulated. The patient was also noted to have mild axillary hidradenitis suppurativa.

Features such as acral pigmentation, palmar pits, rosacea-like eruption, or multiple seborrheic keratoses were not noted.

\section{Results}

Histology of a punch biopsy of one of the pitted scars on the face showed a marked proliferation of elongated and anastomosing, pigmented rete ridges, penetrating into the dermis. A dilated follicular infundibulum filled with basket-weave orthokeratosis was also noted. No acantholysis was observed (Fig. 3).

\section{Discussion/Conclusion}

This case is worthy of note because the secondary findings of the condition, i.e., the comedo-like lesions and pitted scarring, were the most prominent clinical manifestations of the disease, with the pigmentation only being striking in the axillae. Histological examination allowed the diagnosis to be made. Phenotypic variants of DDD have been reported, such as a folliculocentric variant, a vesicular variant, and a variant with prominent hypopigmented macules [1-3]. The coexistence of various dermatological conditions, including hidradenitis 
Fig. 3. Anastomosing pigmented rete ridges surrounding plugged follicular opening. HE. $\times 10$.



suppurativa, has also been well described [4]. Reports of the clinical overlap of various reticulated pigmentation disorders are frequent [4]. In short, significant phenotypic variation has been reported in this group of conditions.

The observation that DDD may present primarily with large comedo-like lesions and pitted scarring rather than pigmentation should prompt dermatologists to consider this group of conditions when entertaining diagnoses such as comedonal acne and the familial dyskeratotic syndromes.

\section{Statement of Ethics}

The authors have no ethical conflicts to disclose. Written consent was obtained from the subject.

\section{Disclosure Statement}

The authors have no conflicts of interest to declare.

\section{Funding Sources}

There were no funding sources.

\section{Author Contributions}

B.M. Tod: wrote the manuscript and performed an administrative role. I. Steenkamp: initial concept and manuscript writing. H.F. Jordaan: initial concept, histological photographs, and editing. W.I. Visser: development of concept and manuscript preparation and editing. 
Tod et al.: Dowling-Degos Disease Presenting Primarily with Comedones and Atrophic Scarring

\section{References}

1 Singh S, Khandpur S, Verma P, Singh M. Follicular Dowling Degos disease: a rare variant of an evolving dermatosis [Internet]. Indian J Dermatol Venereol Leprol. 2013 Nov-Dec;79(6):802-4. Available from: http://www. ijdvl.com/text.asp?2013/79/6/802/120734

2 Linke M, Orouji A, Géraud C. Vesicular variant of Dowling-Degos disease. Br J Dermatol. 2018 Sep;179(3): 795-6.

3 Naveen KN, Athaniker SB, Hegde SP, Shetty R, Radha H, Parinitha SS. Atypical cases of Dowling-Degos disease [Internet]. Indian Dermatol Online J. 2016 Mar-Apr;7(2):99-102. Available from: http://www.idoj.in/text. asp?2016/7/2/99/178096

4 Rathoriya SG, Soni SS, Asati D. Dowling-Degos disease with reticulate acropigmentation of Kitamura: extended spectrum of a single entity [Internet]. Indian Dermatol Online J. 2016 Jan-Feb;7(1):32-5. Available from: http://www.idoj.in/text.asp?2016/7/1/32/174307 